\title{
The characteristics of false negative cervical smears-implications for the UK cervical cancer screening programme
}

\author{
R W Baker, J P O’Sullivan, J Hanley, D V Coleman
}

\begin{abstract}
Objective-To accurately determine whether there are any features of an abnormal cervical smear that predispose to the production of a false negative report, in order to gain insight into why false negative reports are issued, and to establish whether there are steps that can be taken to reduce them.

Design-A quantitative retrospective analysis using the AxioHOME microscope of the number, size, and spatial distribution of abnormal cells in a set of 50 slides comprising a mixture of false negative and true positive cervical smears.

Setting-Five different cytology laboratories in the United Kingdom.

Results-False negative smears were found to be quantitatively different from true positive smears. They contained significantly fewer abnormal cells (median number of abnormal cells for false negatives $=173$, median number of abnormal cells for true positives $=1712$; $p<0.004)$, and these were more likely to be unevenly distributed on the slide. It was possible to predict with a high degree of accuracy whether a smear was a false negative by analysing number and distribution alone $(\kappa=0.57)$.

Conclusions-False negatives are quantitatively different from true positive cervical smears. This has important implications for quality assurance in the UK cervical screening programme. More consideration needs to be given to the effectiveness of existing quality assurance measures, which need to be tailored to the preferential detection of this type of abnormal cervical smear. (F Clin Pathol 1999;52:358-362)
\end{abstract}

Imperial College of

Medicine at St Mary's,

St Mary's Hospital,

Praed Street, London

W2 1PG, UK

$\mathrm{R}$ W Baker

J Hanley

D V Coleman

Department of

Cytopathology, St

Richard's Hospital,

Chichester, West

Sussex, UK

J P O'Sullivan

Correspondence to:

Professor Coleman.

Accepted for publication 15 December 1998
Keywords: cervical cytology; false negative rate

False negative rates in cervical cytology are often quoted as being between $5 \%$ and $50 \%$. The wide variation can be accounted for by different study design and by different methods of calculating the false negative rate. It is extremely difficult to determine the absolute false negative rate accurately; most studies therefore quote relative values, based on the sensitivity of cervical screening against the final cytology report, or against histologically confirmed lesions. The exact scale of the false negative problem is therefore impossible to quantify. Despite this, the problem of false

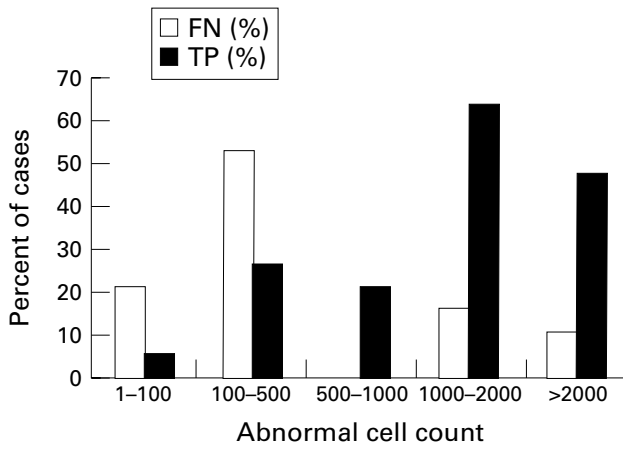

Figure 1 Histogram of cell counts: true positive (TP) versus false negative (FN).

negative cytology has recently resulted in a high level of adverse publicity for certain cytology laboratories.

The reasons why a false negative report might be issued are poorly understood. One method of determining the false negative rate is by review of previous negative cervical smears women with histologically confirmed cases of CIN3 or invasive squamous carcinoma. These smears often contain abnormal cells, and their frequency gives an approximate indication of the laboratory false negative rate. Although this approach is not necessarily of any benefit to the patient, since mistakes only come to light after a long delay, analysis of the cellular content of these slides can give us an insight into the reasons why a false negative report might be generated. In this retrospective study, we have analysed the cellular characteristics of cervical smears with the AxioHOME microscope, which is a system for the quantitative analysis of microscopic variables. The test cohort comprised smears from women with histologically confirmed cases of

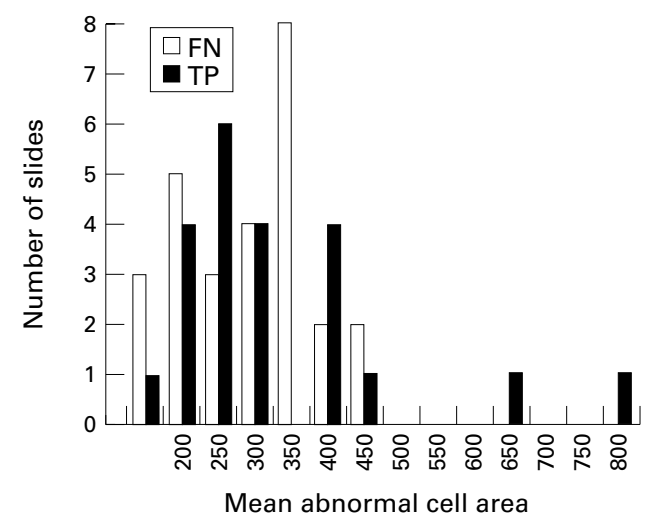

Figure 2 Histogram of mean abnormal cell size: true positive (TP) versus false negative (FN). 

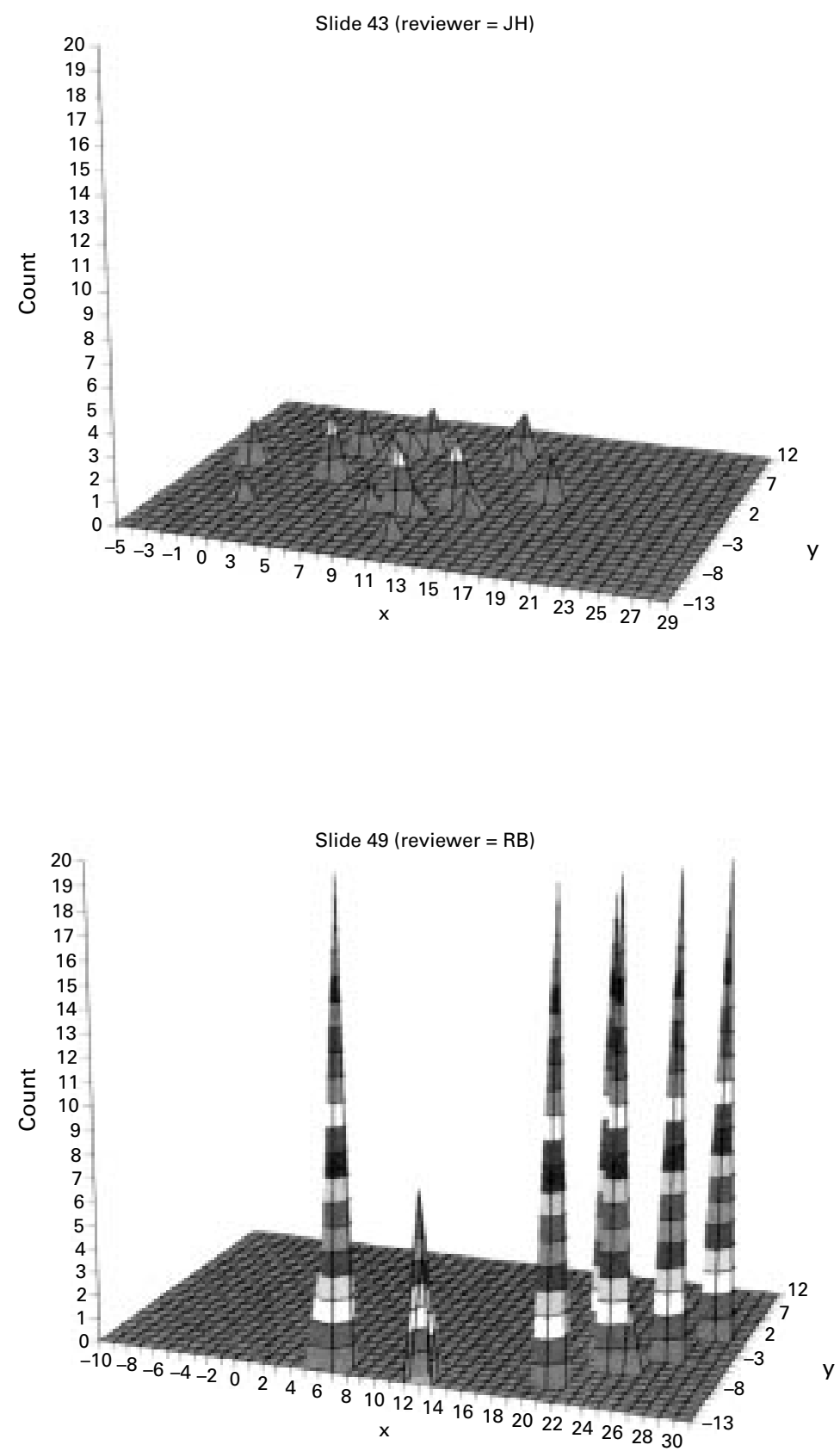

Figure 3 Typical distribution pattern outputs from the AxioHOME microscope for false negative slides. The horizontal axes $x$ and $y$ represent the covered area of the glass slide; the vertical peaks represent counts of abnormal cells in each part of the slide (truncated at 20 cells).

CIN3 which were originally reported as being negative but which on subsequent review were found to contain abnormal cells. True positive smears were admixed as a control set in a randomised, blinded order. The AxioHOME was used to accurately mark all abnormal cells, and to make measurements of the size, number, and distribution of a representative sample of the abnormal cells.

\section{Methods}

TEST COHORT

The test slides comprised 150 cervical smears collected by JPO'S. These were obtained from five cytology laboratories in the United Kingdom, each centre providing 30 smears. The centres each reviewed previously archived negative smears from cases of histologically confirmed CIN3 (cervical intraepithelial neoplasia, grade 3 ). Those smears which contained dyskaryotic cells missed during the original screening process, generating a false negative report, were designated as "false negative" (FN) for the purpose of this study.

The centres then retrieved two abnormal smears reported immediately before and immediately after the false negative smears, with dyskaryotic cells which had been detected during the original screening process, generating a true positive report; these slides were designated "true positive" (TP) slides for the purposes of this study.

Each centre then submitted 30 slides, including $10 \mathrm{FN}$ smears, and $20 \mathrm{TP}$ smears, making a test set of 150 smears, comprising 50 $\mathrm{FN}$ and $100 \mathrm{TP}$ smears.

For this study we included 50 of the set of 150 cervical smears, selected by JPOs in a proportion of FN to TP which was unknown to the reviewers (JH and $\mathrm{RB})$.

AXIOHOME MICROSCOPE

The AxioHOME microscope consists of a computer attached to a microscope which can measure movement on the microscope stage, and projects an image directly onto the field of view. The observer can use the computer mouse to make direct measurements of microscopic objects. The technical specifications of the AxioHOME have been described in detail elsewhere. ${ }^{12}$ The system is extremely useful for the accurate measurement of variables such as cell size and cell count. It has a user based decision making process for measuring morphometric variables, based on the principle of drawing directly around the microscope image with the mouse. This means that it can easily be used in the case of overlapping cells to accurately delineate the boundaries of the cells. However, variation can be introduced through the user's choice of cell to draw, and also through inaccurate drawing of the microscope image. These variables can be reduced through the use of a high quality mouse and through experience in the drawing technique. Both variables were tested by repeating the measurements for a subset of the cases $(n=5)$ by the same reviewer (to measure intraobserver variation) and by a different reviewer (to measure interobserver variation).

The AxioHOME microscope was calibrated before each analysis.

\section{PROTOCOL FOR MEASUREMENTS}

The slides were anonymised, cleaned, and all marks removed. The coordinates of the coverslip were recorded in the general morphometry mode of the AxioHOME microscope.

The slides were then screened using the screening/reviewing software of the AxioHOME, to gain an overall impression of the number and distribution of dyskaryotic cells. This process also ensured that the whole area under the coverslip was observed by the reviewers. The AxioHOME was then placed in the general morphometry mode for the 
accurate determination of the size, number, and distribution of dyskaryotic cells.

The precise location of every dyskaryotic cell in the smear was mapped by placing an "electronic dot" exactly on top of each cell. Where the abnormal cell count exceeded 2000 cells, it was decided to mark representative dyskaryotic cells only up to a count of 2000 , since larger counts slowed down the AxioHOME software and were too labour intensive. Additional cell counts for these very cellular slides were then estimated, and this was added to the number counted quantitatively to give an estimated total for slides with many abnormal cells.

The size of up to 50 single dyskaryotic cells was measured by drawing around the cell perimeter with the mouse.
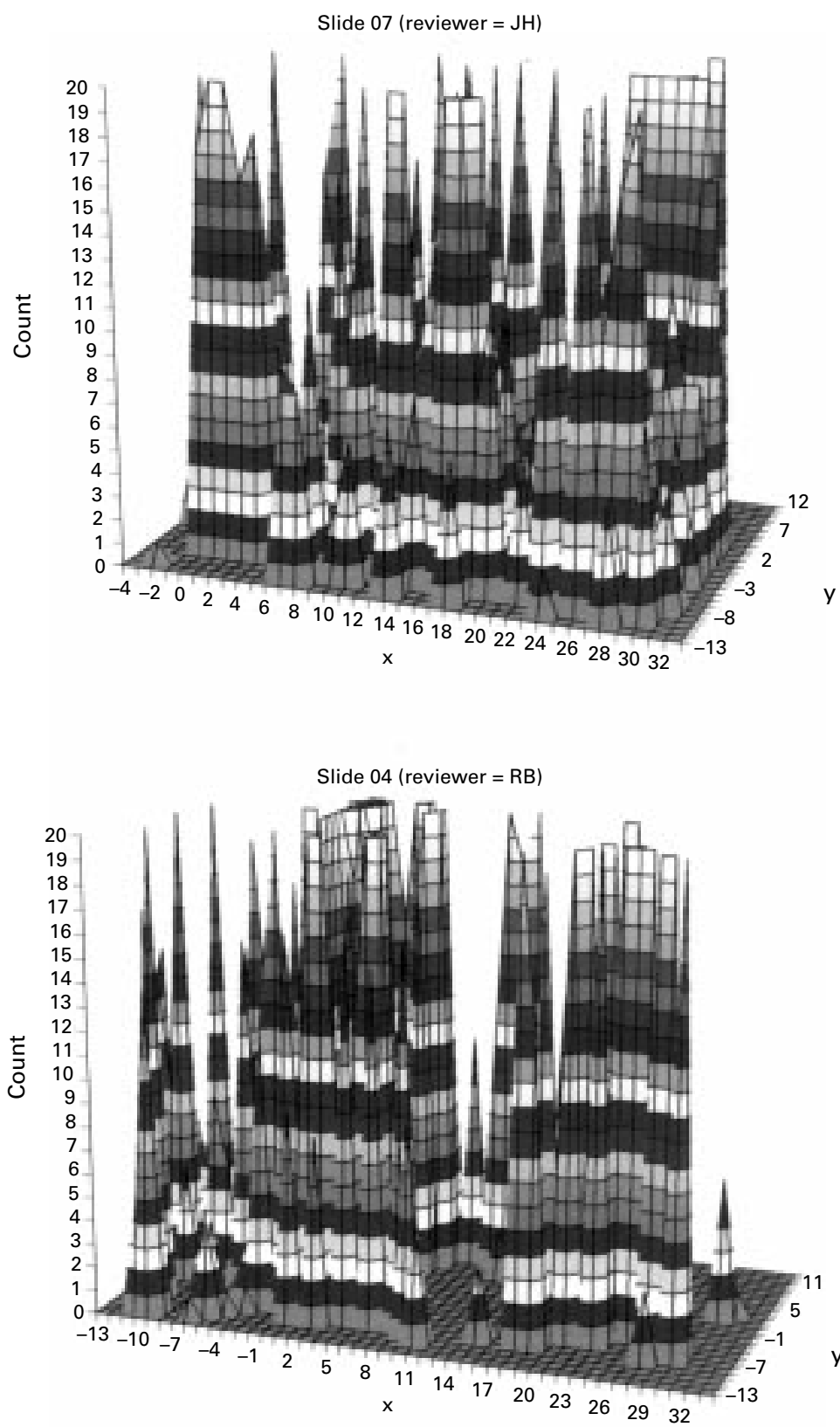

Figure 4 Typical distribution pattern outputs from the AxioHOME microscope for true positive slides. The horizontal axes $x$ and $y$ represent the covered area of the glass slide; the vertical peaks represent counts of abnormal cells in each part of the slide (truncated at 20 cells).
The nucleus to cytoplasm ratio $(\mathrm{n} / \mathrm{c})$ was determined by measuring the nuclear area of the same cells, and dividing the recorded nuclear area by the recorded cell area (cytoplasmic area), to give a precise $\mathrm{n} / \mathrm{c}$ ratio.

The data were exported from the AxioHOME system in dbase IV format into Microsoft Access, where a custom designed database processed the coordinates and produced a distribution table in the form of a grid of $x-y$ coordinates with the cell count of abnormal cells in each square of the grid. This was then processed by Microsoft Excel into a distribution map, to give a clear indication as to the cell distribution for each slide.

Mean cell size and mean $\mathrm{n} / \mathrm{c}$ ratio for each slide was calculated using SPSS statistical package.

The reviewers then predicted whether each slide was an FN or a TP slide by comparing cell counts and distribution maps alone. The actual slide status (TP or FN) was then disclosed and this result was compared with the predicted status of the slide (TP or FN). A $\kappa$ statistic was calculated for the level of agreement of the predicted slide status versus the actual slide status.

\section{Results}

ABNORMAL CELL COUNT

Figure 1 shows the total abnormal cell count for each of the FN slides analysed, and for each of the TP slides analysed. The median abnormal cell count for FN slides was 163. The median abnormal cell count for TP slides was 1712. These results were significantly different $(\mathrm{p}<0.004)$.

\section{ABNORMAL CELL SIZE}

Figure 2 shows the mean abnormal cell size for the FN and TP slides analysed. The mean cell size for $\mathrm{FN}$ slides was $268.4 \mu \mathrm{m}^{2} \quad(95 \%$ confidence interval (CI), 222.0 to 313.9 ). The mean cell size for TP slides was $340.01 \mu^{2}$ (95\% CI, 294.3 to 385.7 ). These results were not significantly different.

ABNORMAL CELL NUCLEUS TO CYTOPLASM RATIO The mean $\mathrm{n} / \mathrm{c}$ ratio for abnormal cells in the $\mathrm{FN}$ slides was 0.379 . The mean $\mathrm{n} / \mathrm{c}$ ratio for abnormal cells in the TP slides was 0.359 . These were not significantly different.

\section{DISTRIBUTION OF ABNORMAL CELLS IN SLIDE}

Figure 3 shows a typical example of the number and distribution of abnormal cells for an FN slide. Figure 4 shows a typical example of the number and distribution of abnormal cells for a TP slide. Figure 5 shows the FN and TP slides classified from the distribution maps into five different categories.

\section{PREDICTION OF SLIDE STATUS}

After measurements were finished, the true status of each slide was disclosed to the reviewers, and a comparison made of the predicted status to the true status of each slide. The status of 41 of 50 slides was predicted correctly, giving a $\kappa$ statistic of 0.57 (95\% CI, 0.30 to 0.84). 


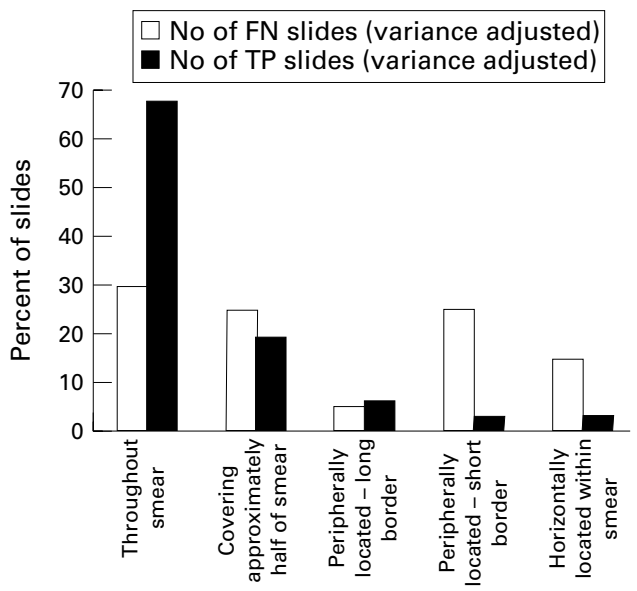

Distribution pattern

Figure 5 Distribution pattern of false negative and true positive slides.

INTRA- AND INTEROBSERVER VARIATION

Intraobserver variation was not significant $(t=0.63, \mathrm{p}=0.563)$.

There was no significant difference in the values for cell counts of independent reviewers. The difference was not significant between reviewers 1 and $2(t=0.36, \mathrm{p}=0.757)$, and between reviewers 2 and $3 \quad(t=-0.10$, $\mathrm{p}=0.927)$.

\section{Discussion}

This study provides the first quantitative evidence that false negative cervical smears are quantitatively different from true positive smears, and that smears with a very few abnormal cells, distributed unevenly on the slide, are much more difficult to diagnose correctly.

From our study it is possible to deduce that certain types of cervical smear are predisposed to the generation of a false negative report. These cases therefore are worthy of special consideration in the quality assurance setting. If these are the most common smears contained in the laboratory false negative fraction, then it is important that any quality assurance method be attuned to the preferential detection of such types of smear. Unfortunately this is not currently the situation in the United Kingdom; existing recommendations for quality assurance are not based on methodology specifically evaluated on the laboratory false negative fraction, but instead concentrate on methods tested on a laboratory backlog. ${ }^{3}$

Furthermore, the low slide coverage and high screening rate associated with these methods ${ }^{5}$ make them unlikely to detect the type of abnormal smear which generates a false negative report.

The results described here suggest that a primary reason for the inability of the human observer to detect the abnormal cells in a smear with a false negative report is to do with the problem of habituation; that is to say, the observer sees many millions of microscopic images which are very similar to the searched for cells, but which are benign and normal in every sense. An example might be small haemopoietic cells. This process of continual exposure desensitises the observer to this type of object, so that on the rare occasion when the image is one of a small, severely dyskaryotic cell, the previous high level of exposure means that the cells are ignored, with disastrous consequences for the woman from whom the smear was obtained.

The study also shows that low abnormal cell counts and small abnormal cells do not account for all instances of $\mathrm{FN}$ reports. We have shown in a previous study ${ }^{6}$ using the AxioHOME to monitor slide coverage and screening time, that primary screeners do not always cover the whole area under the coverslip, and that FNs are likely to arise when slide scanning rates are high. A typical scanning rate for primary screening is 1.7 microscope fields a second, but scanning rates higher than four fields a second are likely to generate false negative reports. These are separate issues, which also have not received the attention that they deserve. These problems may account for those instances of $\mathrm{FN}$ reports where there are abundant or large, well displayed cells.

Our results are similar to those of Bosch et al and Mitchell and Medley, ${ }^{7}$ who used a similar design, except that variables were measured qualitatively, and therefore represent much less accurate data than ours. We did not attempt to measure variables which could not be accurately assessed with the AxioHOME, such as nuclear chromasia.

Our results do not show any statistically significant difference in the size of abnormal cells between false negative and true positive smears, but fig 2 suggests that the results are tantalisingly close to showing a significant difference, which perhaps will only be demonstrated by another study on a larger sample.

Our study has also showed the versatility of the AxioHOME system in the morphometric and spatial analysis of cytological objects. Inter- and intraobserver variations are relatively small, and the system provides output data that can be easily manipulated, even by custom designed software.

This study highlights the need for a reevaluation of the processes involved in the screening of cervical smears in the cytology laboratory, and continuing investigation of the characteristics of false negative smears. Such research may eventually reward us with a better insight into the reasons why false negative smears are generated, and a deeper understanding of how these tragic events can be minimised.

We would like to thank the pathologists from the participating centres for their patience and willingness to loan us the smears which made up the cohort for this study. These are Dr A Al-Naffusi, Royal Infirmary, Edinburgh, Dr MT Brett, Southmead Hospital, Bristol, Dr A Herbert, Southampton General Hospital, Dr ME McKean, Aberdeen Royal Infirmary, and Dr CA Waddell, Birmingham Women's Hospital. We would also like to thank Mr Moray Fleming for assistance in the production of the software for analysing the AxioHOME output, and $\mathrm{Mr}$ of the software for analysing the AxioHOME output, and $\mathrm{Mr}$ Barry Leete of Zeiss UK for advice and support. This research SOC 94/202239 05F02: ITEM B34304.

1 Brugal G, Dye R, Krief B, et al. HOME: Highly Optimised Microscope Environment. Cytometry 1992;13:109-16. 2 Morens A, Krief, B, Brugal G. Tutorial: the HOME Microscope Workstation. Anal Quant Cytol Histol 1992;14:28993. 
3 Baker A, Melcher DH. Rapid cervical cytology screening. Cytopathology 1991;2:299-301.

C Faraker CA. Partial irescreening o improved method of quality assurance in laboratories undertaking cervical screening. Cytopathology 1993;4:8595.

5 Baker RW, Wadsworth J, Brugal G, et al. An evaluation of "rapid review" as a method of quality control of cervical smears using the AxioHOME microscope. Cytopathology 1997;8:85-95.
6 Baker RW, Brugal G, Coleman DV. Assessing slide coverage by cytoscreeners during the primary screening of cervical AxioHOME microscope system. Anal Cell Pathol 1997;13:29-37.

7 Bosch MMC, Rietweld-Scheffers PEM, Boon ME. Characteristics of false-negative smears tested in the normal screening situation. Acta Cytol 1992;36:711-16.

8 Mitchell H, Medley G. Differences between Papanicolaou smears with correct and incorrect diagnoses. Cytopathology 1995;6368-75.

\section{Fournal of Clinical Pathology - http://www.jclinpath.com}

Visitors to the world wide web can now access the fournal of Clinical Pathology either through the BMJ Publishing Group's home page (http://www.bmjpg.com) or directly by using its individual URL (http://www.jclinpath.com). There they will find the following:

- Current contents list for the journal

- Contents lists of previous issues

- Members of the editorial board

- Information for subscribers

- Instructions for authors

- Details of reprint services.

A hotlink gives access to:

- BMJ Publishing Group home page

- British Medical Association web site

- Online books catalogue

- BMJ Publishing Group books.

The web site is at a preliminary stage and there are plans to develop it into a more sophisticated site. Suggestions from visitors about features they would like to see are welcomed. They can be left via the opening page of the BMJ Publishing Group site or, alternatively, via the journal page, through "about this site". 